\title{
The Camouflage Evaluation Model Based on Fuzzy Comprehensive Evaluation
}

\author{
Rongrong Cai \\ Department of Economics and Management of North China Electric \\ Power University, Baoding,071000, China
}

\begin{abstract}
This paper whose core thought is fuzzy comprehensive evaluation mainly studies the camouflage effects of different camouflage patterns, establishes a camouflage effect evaluation system by analyzing the sample data and explores the relationship between the effect of camouflage and some indexes. We introduce fuzzy comprehensive evaluation method to establish a model to evaluate the camouflage effects of various camouflage patterns after considering that the problem is fuzzy and difficult to quantify. In order to make the evaluation system more objective, this paper uses the entropy method, and then quantifies the index according to the sample data and determines the corresponding weights.

Keywords:Fuzzy Comprehensive Evaluation; K-Means Clustering Technique; Canny Edge Detection; Camouflage Evaluation

\section{Introduction}

This section mostly analyzes the low-visibility effect of various patterns and develops a quantitative camouflage effect evaluation method. There are many factors affecting the accuracy and objectivity of the low-visibility effect of camouflage, Therefore, to objectively assess the effectiveness of camouflage pattern ,we have adopted fuzzy Comprehensive Evaluation repeatedly to evaluate the effectiveness of a camouflage pattern of random to some special kinds of terrain, then get a final result by average weighting method. In this paper, a fuzzy comprehensive evaluation method which can greatly reduce enough human and material resources and improve work efficiency is used to assess a pattern camouflage pattern.
\end{abstract}

\section{Modeling: The FCE for Camouflage Patterns}

\subsection{Establishing the Factors Set}

Currently, there are some main factors affecting the camouflage effect, such as the contrast of background brightness between the camouflage pattern with the original, surface characteristics, atmospheric visibility and exposure to size and so on. From above, we can easily establish the following factors set. 


\subsection{The factor set of brightness contrast: $U_{1}$}

Brightness is the most direct stimulation of the eye contact. If the Brightness camouflage pattern is similar to the original background, we can prove it has a low-visibility and a good camouflage effect .On the contrary, if the brightness contrast is high, it would be easy to cause the attention of the enemy with a poor camouflage effect.

\subsubsection{The factor set of texture : $U_{2}$}

Texture is an important feature and can clearly reflect the homogeneity Phenomenon.[1]

This paper introduces Gray Level Co-occurrence Matrix, GLCM, for short, to do the texture analysis. This paper selects four kinds of typical secondary statistics to characterize the texture, they are moment of the inertia $\mathrm{E}$, contrast ratio I, entropy $\mathrm{H}$, and Relevance C.by calculating the eigenvalues, we obtain the texture feature vector $\mathrm{T}=(\mathrm{E}, \mathrm{I}, \mathrm{H}, \mathrm{C})$.Next, we introduce Gaussian normalization to Solve the problem of the big difference between some eigenvalue. Four mean eigenvalues $\mathrm{m}$ and standard deviation $\sigma$ are calculated. And then reuse Eq. 1 where in the charac[2]teristic value normalized to $[0,1]$.

$$
f_{i}^{\prime}=\left(\frac{f_{i}-m}{3 \sigma}+1\right) / 2 .
$$

\subsubsection{The factor set of color characteristics: $U_{3}$}

2.2.3. The factor set of Reflection characteristics: $U_{4}$.

\subsubsection{Establishing the Weight Set}

Many factors make a different to the effect of camouflage, and the importance of each factor is basically not the same, so it is necessary to attach a weight to each factor. According to the importance of each level of each index. We assign every factor with corresponding weighting factor. We adopt entropy method to calculate the weights. If the information entropy index is smaller, the greater the amount of information provided by this indicator, in the comprehensive evaluation of the role they should be bigger, the weight should be higher.

- Data Standardization

If the number of index is $k$,

$$
X_{i}=\left\{x_{1}, x_{2}, \ldots, x_{n}\right\}
$$

Then

$$
Y_{\mathrm{ij}}=\frac{X_{i j}-\min \left(X_{i}\right)}{\max \left(X_{i}\right)-\min (X i)}
$$

Where $X_{i}$ stands for each index, and $Y_{\mathrm{ij}}$ refers to the value after data standardization. 
- Finding the Entropy of Each Index

According to the definition of entropy in information theory, a set of information entropy data is

$$
E_{j}=-\ln (n)^{-1} \sum_{i=1}^{n} p_{i j} \ln p_{i j}(\mathrm{j}=1,2, \ldots, \mathrm{k})
$$

Where

$$
p_{i j}=\frac{Y_{i j}}{\sum_{i=1}^{n} Y_{i j}}
$$

If $p_{i j}=0$, then $\lim _{p_{i j} \rightarrow \infty} p_{i j} \ln p_{i j}=0$.

- Determining the weight of each index

According to the equation of entropy formula, work out the results of information entropy of each index $\left(E_{1}, E_{2} \ldots, E_{k}\right)$, then get the weight of each index through the next equation[3]

$$
W_{i}=\frac{1-E_{i}}{k-\sum E_{i}}(\mathrm{i}=1,2, \ldots, k) .
$$

\subsubsection{Establishing the Grade Evaluation Set}

Grade evaluation set for this model are $=\{$ Disappointing, Poor , General Good , Outstanding

We apply triangular fuzzy number to process the scores to get their respective grade and get the result as follow:

\begin{tabular}{|l|c|c|l|c|l|}
\hline Rank & Disappointing & Poor & General & Good & Outstanding \\
\hline Scores & $(50,50,60)$ & $(50,60,70)$ & $(60,70,80)$ & $(70,80,90)$ & $(80,90,90)$ \\
\hline
\end{tabular}

2.2.6. The Application

Taking woodland show in Fig 2) for example. Firstly, we selected eight different camouflage pattern (show in Fig 1) and respectively calculate their camouflage effect in this terrain with brightness contrast, texture, color characteristics, and reflection characteristics as the indicator. Secondly we apply the same method again to calculate the effect which eight camouflage patterns generate to other terrains such as snow and desert. In this way, we finally analyze the low-visibility effect of various patterns, and develop a quantitative camouflage effect evaluation method. 


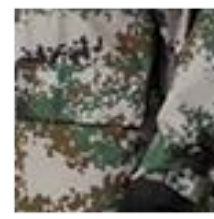

camouflage 1

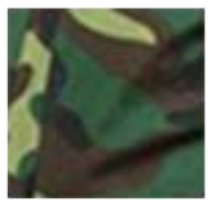

camouflage 5

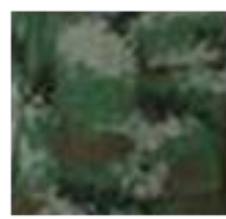

camouflage 2

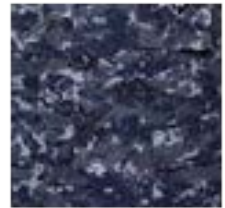

camouflage 6

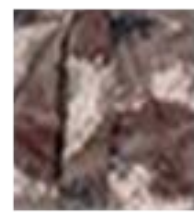

camouflage 3

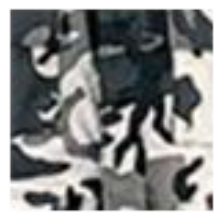

camouflage 7

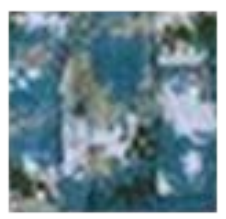

camouflage 4

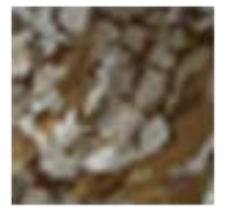

camouflage 8

Fig.1

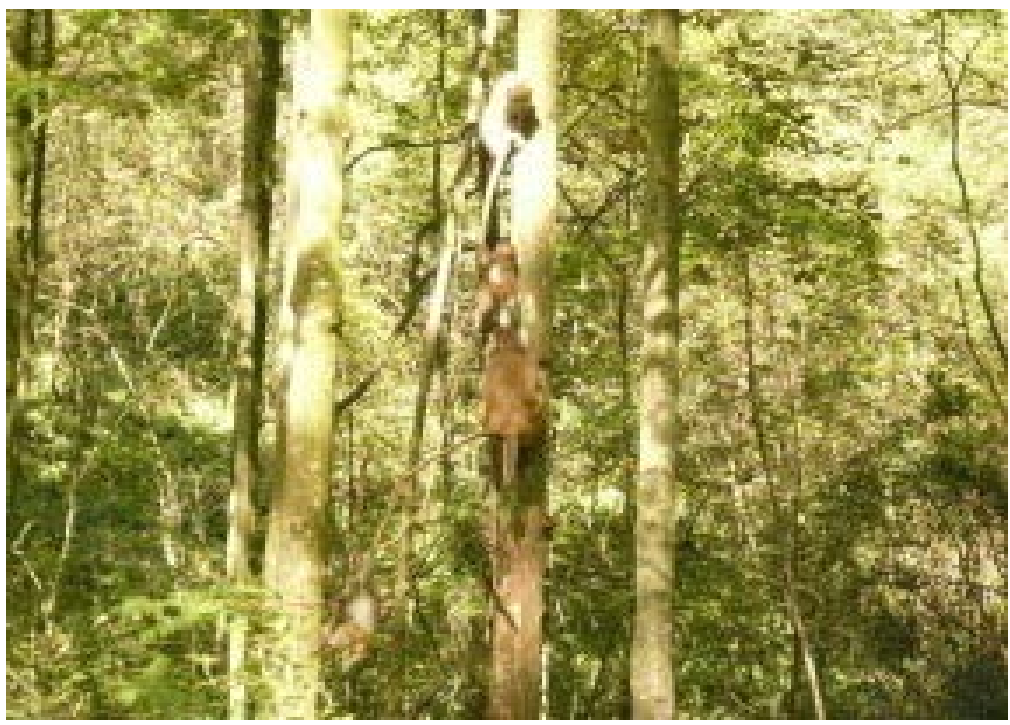

Fig. 2

Then extract quantized color histogram of the background color and pattern of camouflage $1 \sim 8$, and obtain the color similarity with the background image between camouflage $1 \sim 8$, they are $0.037,0.078,0.016,0.121,0.027,0.162$, 0.049, 0.009 .

Extract the GLCM of the background and camouflage 1 8 image respectively and the Statistics its four eigenvalues E, I, H and C, and normalized. The results are shown in Table. 2. 
Table. 2

\begin{tabular}{|c|c|c|c|c|c|}
\hline & texure:U2 & $0^{\circ}$ & $45^{\circ}$ & $90^{\circ}$ & $135^{\circ}$ \\
\cline { 2 - 6 } background & $\mathrm{E}$ & 0.018504 & 0.015296 & 0.01677 & 0.01727 \\
\cline { 2 - 6 } & $\mathrm{H}$ & 4.334166 & 4.530222 & 4.43841 & 4.414681 \\
\cline { 2 - 6 } & $\mathrm{I}$ & 4.129737 & 7.12214 & 5.385027 & 5.11935 \\
\cline { 2 - 6 } & $\mathrm{C}$ & 0.107038 & 0.068913 & 0.091008 & 0.094371 \\
\hline \multirow{5}{*}{ camouflage } & texure:U2 & $0^{\circ}$ & $45^{\circ}$ & $90^{\circ}$ & $135^{\circ}$ \\
\cline { 2 - 6 } & $\mathrm{E}$ & 0.02252 & 0.018036 & 0.022699 & 0.018313 \\
\cline { 2 - 6 } & $\mathrm{H}$ & 4.389273 & 4.531069 & 4.386773 & 4.522279 \\
\cline { 2 - 6 } & $\mathrm{I}$ & 8.810162 & 12.81158 & 8.722687 & 12.47337 \\
\cline { 2 - 6 } & $\mathrm{C}$ & 0.051361 & 0.039026 & 0.051588 & 0.040066 \\
\hline
\end{tabular}

Utilize MATLAB to get the result of HSV, shown in Fig. 3.

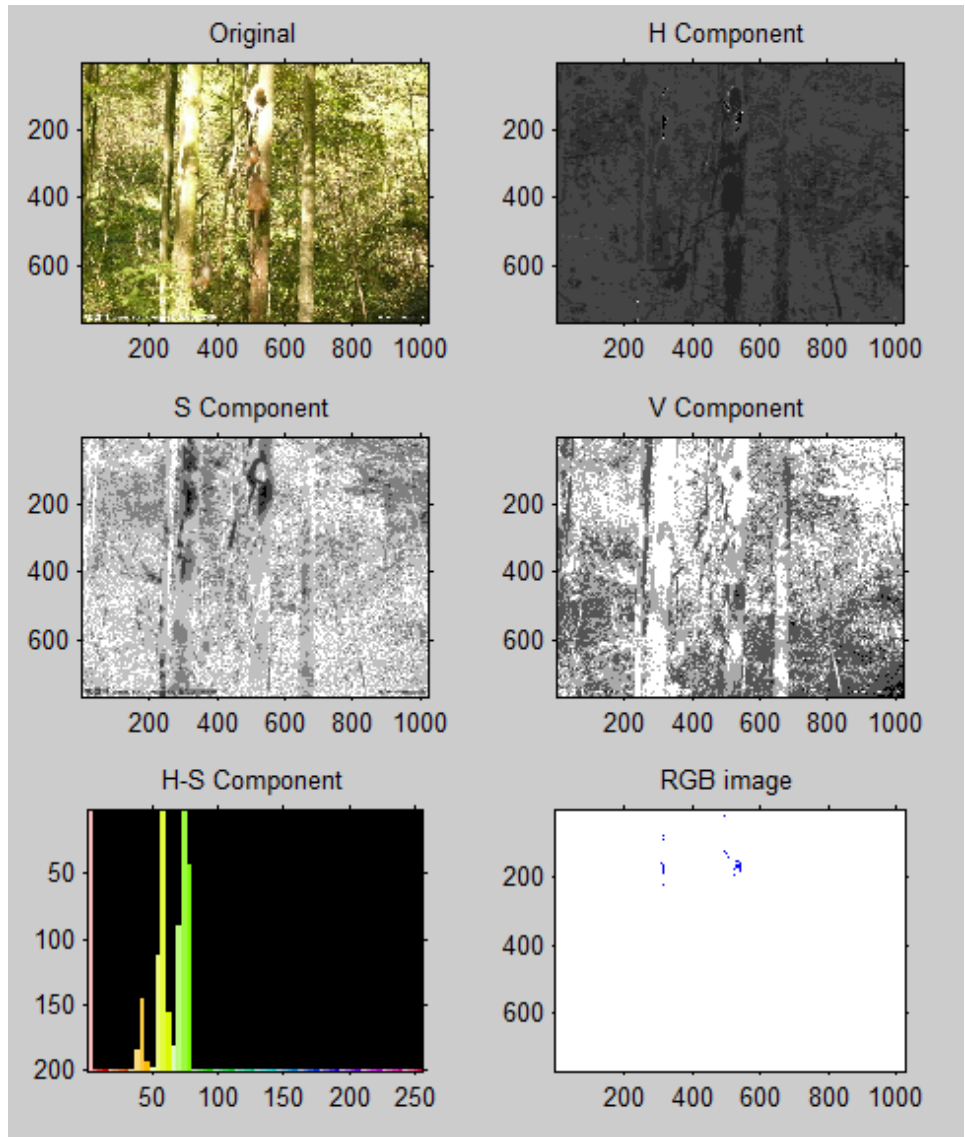

Fig. 3 get the result of HSV 
Induce the method of Manhattan distance to calculate the texture similarity of camouflage $1 \sim 8$.they are $1.4309,1.4154,1.4118,1.4140,1.4401,1.4216,1.4112$, and 1.4120 .

The original dates for the entropy method are shown in Table.3

Table.3 The original dates for the entropy method

\begin{tabular}{|c|c|c|c|c|}
\hline & $\mathrm{U} 1$ & $\mathrm{U} 2$ & $\mathrm{U} 3$ & $\mathrm{U} 4$ \\
\hline Camouflage 1 & 0.1872 & 1.4309 & 0.037 & 0.212 \\
\hline Camouflage 2 & 0.4142 & 1.4154 & 0.078 & 0.431 \\
\hline Camouflage 3 & 0.3442 & 1.4118 & 0.016 & 0.322 \\
\hline Camouflage 4 & 0.4151 & 1.4140 & 0.121 & 0.417 \\
\hline Camouflage 5 & 0.2905 & 1.4401 & 0.027 & 0.589 \\
\hline Camouflage 6 & 0.1753 & 1.4216 & 0.162 & 0.109 \\
\hline Camouflage 7 & 0.2246 & 1.4112 & 0.049 & 0.193 \\
\hline Camouflage 8 & 0.3574 & 1.4120 & 0.009 & 0.307 \\
\hline
\end{tabular}

Utilize the Eq. 6 we can get the weight set, shown in Table.4

Table.4 The weight set

\begin{tabular}{|c|c|c|c|}
\hline W1 & W2 & W3 & W4 \\
\hline 0.1775 & 0.0005 & 0.6577 & 0.1642 \\
\hline
\end{tabular}

$\mathrm{A}=\{\mathrm{W} 1, \mathrm{~W} 2, \mathrm{~W} 3, \mathrm{~W} 4\}=(0.1775,0.0005,0.6577,0.1642)$.

By combining expert investigation and the difference between camouflage pattern and background, we mark the four factors in the way of hundred -mark system and convert them into fuzzy membership degree. [5]The original score table are list in Table.5.

Table. 5 The original score

\begin{tabular}{|l|l|l|l|l|}
\hline & $\mathrm{U}_{1}$ & $\mathrm{U}_{2}$ & $\mathrm{U}_{3}$ & $\mathrm{U}_{4}$ \\
\hline Camouflage 1 & 88 & 85 & 89 & 85 \\
\hline Camouflage 2 & 92 & 87 & 93 & 93 \\
\hline Camouflage 3 & 84 & 89 & 83 & 89 \\
\hline Camouflage 4 & 78 & 91 & 75 & 78 \\
\hline Camouflage 5 & 90 & 79 & 92 & 91 \\
\hline Camouflage 6 & 81 & 93 & 77 & 82 \\
\hline Camouflage 7 & 75 & 80 & 79 & 73 \\
\hline Camouflage 8 & 86 & 83 & 90 & 88 \\
\hline
\end{tabular}

Taking camouflage 6 for example, we apply triangular fuzzy number to process the scores obtained
$\left(\begin{array}{llll}R_{11} & R_{12} & R_{13} & R_{14}\end{array}\right.$
$\left.R_{15}\right)=\left(\begin{array}{llll}0 & 0 & 0 & 0.9\end{array}\right.$
0.1) after 
normalization, which means the evaluation vector of brightness contrast is

$$
\left(\begin{array}{lllll}
0 & 0 & 0 & 0.9 & 0.1
\end{array}\right) \text {. }
$$

We can get the evaluation vector of the other three factors and get the fuzzy relationship matrix, the result is shown in

$$
R=\left(\begin{array}{ccccc}
0 & 0 & 0 & 0.9 & 0.1 \\
0 & 0 & 0 & 0 & 1 \\
0 & 0 & 0.7 & 0.3 & 0 \\
0 & 0 & 0 & 0.2 & 0.8
\end{array}\right)
$$

Considering the data of samples is simple, we introduce the method of $M(\bullet, \oplus)$ operator.[4]

$$
B_{\mathrm{j}}=\sum_{i=1}^{n}\left(a_{i}, r_{i j}\right)=\left(a_{i}, r_{i j}\right) \oplus \cdots \oplus\left(a_{i}, r_{i j}\right) \wedge 1
$$

And the result is

$$
\begin{aligned}
B=A \circ R & =(0.1775,0.0005,0.6577,0.1642) \circ\left(\begin{array}{ccccc}
0 & 0 & 0 & 0.9 & 0.1 \\
0 & 0 & 0 & 0 & 1 \\
0 & 0 & 0.7 & 0.3 & 0 \\
0 & 0 & 0 & 0.2 & 0.8
\end{array}\right) \\
& =(0,0,0.46039,0.3899,0.14961)
\end{aligned}
$$

\section{Conclusion}

$$
B=A \circ R=(0,0,0.46039,0.3899,0.14961)
$$

Means the camouflage effect of camouflage 6 belongs to the third grade, that is to say the camouflage effect of it is general. We can get the camouflage effect of other patterns. Here are the best four patterns in three different terrains(in descending order of priority),shown in Table 6.

Table. 6 best four patterns in three different terrains(in descending order of

$$
\text { priority) }
$$

\begin{tabular}{|c|c|c|c|c|}
\hline Woodland & Camouflage 2 & Camouflage 5 & Camouflage 8 & Camouflage 1 \\
\hline Desert & Camouflage 8 & Camouflage 1 & Camouflage 3 & Camouflage 7 \\
\hline Snow & Camouflage 7 & Camouflage 8 & Camouflage 1 & Camouflage 4 \\
\hline
\end{tabular}




\section{References}

[1] Entropy method [EB/OL].

http://baike.baidu.com/view/2466479.htm?fr=aladdin.

[2]Bashar Bashar M K, Matsumoto T, Ohnishi N. Wavelet transform- based locally order less images for texture segmentation $[\mathrm{J}]$. Pattern Recognition Letters, 2003, 24 (15): $2633-2650$.

[3] http://blog.sina.com.cn/s/blog_710e9b550101aqnv.html [EB/OL]. Using entropy to determine the weight of index.

[4] Li Yulin ,Gao Zhigang ,Han Yanling The Determination of Weight Value and the Choice of Composite Operator s in Fuzzy Comprehensive Evaluation[J] Computer Engineering and Applications, 2006,23:38-42+197

[5]S.P. Adhau, R.M. Moharil, P.G. Adhau K-Means clustering technique applied to availability of micro hydro power [C] Sustainable Energy Technologies and Assessments,2014 